\title{
One- and two-phonon capture processes in quantum dots
}

\author{
Magnúsdóttir, Ingibjörg; Uskov, Alexander; Bischoff, Svend; Tromborg, Bjarne; Mørk, Jesper
}

Published in:

Journal of Applied Physics

Link to article, DOI:

$10.1063 / 1.1512694$

Publication date:

2002

Document Version

Publisher's PDF, also known as Version of record

Link back to DTU Orbit

Citation (APA):

Magnúsdóttir, I., Uskov, A., Bischoff, S., Tromborg, B., \& Mørk, J. (2002). One- and two-phonon capture

processes in quantum dots. Journal of Applied Physics, 92(10), 5982-5990. https://doi.org/10.1063/1.1512694

\section{General rights}

Copyright and moral rights for the publications made accessible in the public portal are retained by the authors and/or other copyright owners and it is a condition of accessing publications that users recognise and abide by the legal requirements associated with these rights.

- Users may download and print one copy of any publication from the public portal for the purpose of private study or research.

- You may not further distribute the material or use it for any profit-making activity or commercial gain

- You may freely distribute the URL identifying the publication in the public portal

If you believe that this document breaches copyright please contact us providing details, and we will remove access to the work immediately and investigate your claim 


\title{
One- and two-phonon capture processes in quantum dots
}

\author{
I. Magnusdottir, ${ }^{\text {a) }}$ A. V. Uskov, ${ }^{\text {b) }}$ S. Bischoff, B. Tromborg, and J. Mørk \\ COM, Technical University of Denmark, Bldg. 345w, DK-2800 Kgs., Lyngby, Denmark
}

(Received 10 July 2002; accepted 12 August 2002)

Multiphonon capture processes are investigated theoretically and found to contribute efficiently to the carrier injection into quantum dots. It is shown that two-phonon capture contributes where single-phonon capture is energetically inhibited and can lead to electron capture times of a few picoseconds at room temperature and carrier densities of $10^{17} \mathrm{~cm}^{-3}$ in the barrier. (C) 2002 American Institute of Physics. [DOI: 10.1063/1.1512694]

\section{INTRODUCTION}

Quantum-dot (QD) lasers with record low-threshold currents have now been realized in several laboratories ${ }^{1-3}$ with self-assembled QDs. Carriers in such lasers are electrically pumped into layers around the dots. Thereafter, they are captured into the dots and relax via the excited QD energy levels to the lower-lying lasing levels. The properties of such lasers thus rely on efficient carrier feeding to the active QD states. The processes involved, carrier capture into the dots and intradot relaxation, have been under extensive research during the past decade, both experimentally and theoretically. ${ }^{4-15}$ Two different capture and relaxation processes have been considered; via carrier-carrier interaction (Auger processes) $)^{5,6,13,14}$ or via carrier-phonon coupling. ${ }^{6,710-12}$ In Ref. 7, capture of a carrier from the wetting layer (WL) to the first excited state in a cone-shaped dot, via emission of one longitudinal optical (LO) phonon, is found to exhibit strong resonances versus dot size. At resonance, the process is shown to be efficient, leading to short capture times in the picosecond range. In this article, we investigate capture of carriers from states in the continuous bulk energy spectrum into the discrete states of QDs via emission of one or two LO phonons. Expressions for the single- and two-phonon capture rates will be derived. Two-phonon capture will be shown to be efficient for states lying too deep for carriers to be captured in a single-phonon process and we will study the role of the different intermediate states in this kind of capture. We will show that two-phonon capture processes can lead to electron capture times of a few picoseconds at room temperature and carrier densities of $10^{17} \mathrm{~cm}^{-3}$ in the barrier. We are not aware of any other investigations of two LO-phonon mediated capture processes in QDs.

\section{MODEL}

The capture of carriers (electrons or holes) from threedimensional (3D) states in the continuous part of the energy spectrum into discrete QD states via emission of one or two phonons can be described by a set of rate equations. ${ }^{16}$ We assume that all the QDs are identical, each containing a

\footnotetext{
a) Electronic mail: im@com.dtu.dk

${ }^{b)}$ Also at Lebedev Physical Institute, Leninsky prospect 53, 117924 Moscow, Russia.
}

single energy level. The rate equations for the occupation probability, $\rho$, of a single QD state and the 3D carrier density, $n$, are then written as

$$
\begin{aligned}
& \frac{d \rho}{d t}=R(1-\rho), \\
& \frac{d n}{d t}=-2 R N_{D}(1-\rho),
\end{aligned}
$$

where $N_{D}$ is the number of quantum dots per unit volume. For $\rho \ll 1$, the rate $R$ gives the mean number of carriers entering the QD state per unit time. It can be measured, in particular, in time-resolved photoluminescence experiments the photoluminescence rise time, $\tau=1 / R$. Below, $\tau$ will be denoted the capture time. The factor of 2 in Eq. (2) is due to spin degeneracy of the QD level. In Eqs. (1) and (2), spontaneous recombination is neglected for simplicity as it is expected to take place on a much longer time scale, and only terms involving the capture process are included. Equation (1) can also be expressed in terms of the QD carrier density, $n_{\mathrm{QD}}=2 N_{D} \rho$ (factor 2 due to spin), as

$$
\frac{d n_{\mathrm{QD}}}{d t}=2 R N_{D}(1-\rho) .
$$

We will show later in this article that for low to moderate 3D carrier densities $n$, the rate $R$ is, to a good approximation, proportional to $n$ :

$$
R=\frac{A}{2} n,
$$

where $A$ is independent of $n$. In this case, Eq. (2) becomes

$$
\begin{aligned}
& \frac{d n}{d t}=-A N_{D} n(1-\rho), \\
& \frac{d n}{d t}=-\frac{n}{\tau_{c}}(1-\rho) .
\end{aligned}
$$

In Eq. (6), we have defined the effective capture time

$$
\frac{1}{\tau_{c}}=A N_{D} .
$$

It is important to note that $\tau_{c}$ depends on the 3D density of dots, $N_{D}$, whereas $\tau$ is the property of a single dot, i.e., it is independent of $N_{D}$. The effective capture time $\tau_{c}$ is an es- 


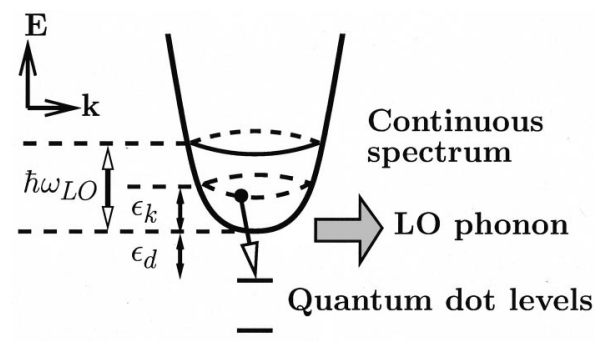

FIG. 1. Single-phonon capture process. A carrier in the continuous part of the spectrum is captured into a QD energy state, thereby emitting one LO phonon of energy $\hbar \omega_{\mathrm{LO}}$. Energy of the incident carrier is denoted by $\varepsilon_{k}$ and the binding energy of the QD state is $\varepsilon_{d}$.

sential parameter in laser dynamics, e.g., through nonlinear gain. ${ }^{15,17}$ In the following sections, we will derive expressions for the capture time $\tau$ and estimate the effective capture time $\tau_{c}$ in single- and two-phonon capture processes. In general, the rate of carrier capture into an empty QD state of definite spin can be written as

$$
R=\sum_{\mathbf{k}} w(\mathbf{k}) f\left(\varepsilon_{\mathbf{k}}\right)
$$

where $w(\mathbf{k})$ is the probability per unit time that the transition will take place for a carrier in the continuous part of the energy spectrum, k being the carrier wave vector. $f$ is the Fermi distribution of the thermalized electron distribution and $\varepsilon_{\mathbf{k}}$ is the energy of the incident carrier.

\section{A. Single-phonon capture}

The single-phonon-mediated capture process (with emission of one LO phonon) is illustrated schematically in Fig. 1. The probability that the carrier is captured into a QD state by emitting a phonon of wave vector $\mathbf{q}$ can be expressed with Fermi's Golden Rule:

$$
w(\mathbf{k}, \mathbf{q})=\frac{2 \pi}{\hbar}\left|\hat{V}_{\mathrm{fi}}^{\mathrm{em}}(\mathbf{q})\right|^{2} \delta\left(E_{f}-E_{i}\right),
$$

where $E_{i}\left(E_{f}\right)$ is the energy of the initial (final) state, $\mathbf{k}$ is the wave vector characterizing the carrier state in the continuous spectrum, $\hat{V}^{\mathrm{em}}$ is the Hamiltonian for phonon emission and $\hat{V}_{\mathrm{fi}}^{\mathrm{em}}(\mathbf{q})$ is the matrix element of $\hat{V}^{\mathrm{em}}$ between the initial and final state. It has been shown ${ }^{8,18}$ that carriers in discrete QD states can couple strongly to LO phonons, forming polarons. The formation of polarons can strongly affect carrier relaxation in QDs. This analysis has been extended to the interaction of carriers in quasibound states with LO phonons ${ }^{19}$ where we have shown that polarons can also be formed with carriers in quasibound states. However, in this article, we consider carrier capture in the framework of standard perturbation theory ${ }^{20}$ and neglect the influence of quasibound states on the carrier capture, so that the initial carrier states in the continuous spectrum are described with plane waves (see below). ${ }^{5,7,13}$ We use a bulk model for the phonon modes, although the phonon spectrum and phonon modes in semiconductor nanostructures can be modified in comparison with bulk semiconductors. For instance, it was shown ${ }^{21}$ that the LO-phonon modes in CdSe/ZnSe QD structures are of mixed character, localized in the QD and extended along a two-dimensional (2D) remnant layer (RL). The detailed consideration of phonon modes in QD structures can be necessary, in particular, for a theoretical explanation ${ }^{21}$ of resonance features in the PL spectra at low temperatures, which are attributed to carriers in the RL in an excitonic state of definite discrete energy. In this article, we concentrate on QD structures (GaAs/AlGaAs, ${ }^{22}$ for instance, and QD laser structures $\left.{ }^{1-3}\right)$, where the material characteristics that define the spatial distribution of phonon modes do not differ significantly in the QD and the barrier material. For such structures, the bulk model for phonons has provided a good description of dephasing rates, ${ }^{22,23}$ polaron effects, ${ }^{24}$ and capture processes. ${ }^{7}$ Note, also, that LO-phonon carrier relaxation rates in quantum wells do not differ significantly, when calculated by either using bulk or confined LO phonons. ${ }^{25}$ Furthermore, we are mainly interested in the carrier dynamics of QD lasers at room temperature. Therefore, we neglect also excitonic effects of carriers in the continuous spectrum. The Hamiltonian for the carrier-LO phonon interaction is given by $^{26}$

$$
\hat{V}=\sum_{\mathbf{q}}\left(\hat{V}_{\mathbf{q}}^{\mathrm{abs}}+\hat{V}_{\mathbf{q}}^{\mathrm{em}}\right)
$$

where $\hat{V}_{\mathbf{q}}^{\mathrm{abs}}$ and $\hat{V}_{\mathbf{q}}^{\mathrm{em}}$ correspond to phonon absorption and emission, respectively. They are given by

$$
\begin{aligned}
& \hat{V}_{\mathbf{q}}^{\mathrm{abs}}=C_{q} e^{i \mathbf{q} \cdot \mathbf{r}} \hat{a}_{\mathbf{q}} \\
& \hat{V}_{\mathbf{q}}^{\mathrm{em}}=C_{q}^{*} e^{-i \mathbf{q} \cdot \mathbf{r}} \hat{a}_{\mathbf{q}}^{\dagger},
\end{aligned}
$$

where $C_{q}$ are the polar coupling coefficients for carrier-LO phonon interaction and $\hat{a}_{\mathbf{q}}\left(\hat{a}_{q}^{\dagger}\right)$ is the operator of annihilation (creation) of an LO phonon with wave vector q. The coupling coefficient is given by

$$
\left|C_{q}\right|^{2}=\frac{\hbar \omega_{\mathrm{LO}} e^{2}}{2 \varepsilon_{0} \varepsilon_{r} \Omega q^{2}},
$$

where $\hbar \omega_{\mathrm{LO}}$ is the LO phonon energy, $e$ is the electronic charge, $\Omega$ is the normalization volume, $\varepsilon_{0}$ the permittivity constant, and

$$
\frac{1}{\varepsilon_{r}}=\frac{1}{\varepsilon_{\infty}}-\frac{1}{\varepsilon},
$$

where $\varepsilon_{\infty}(\varepsilon)$ is the high-frequency (static) dielectric constant. Note that spin is conserved in a phonon-capture process. Therefore, Eq. (8) does not contain a sum over spins.

In this single-phonon capture process, the initial and final phonon states differ only in the number of phonons in mode $\mathbf{q}$

$$
n_{\mathbf{q}}^{f}=n_{\mathbf{q}}^{i}+1
$$

so that

$$
\begin{aligned}
w(\mathbf{k}, \mathbf{q})= & \frac{2 \pi}{\hbar}(\bar{n}+1)\left|C_{q}\right|^{2}\left|\left\langle d\left|e^{-i \mathbf{q} \cdot \mathbf{r}}\right| \mathbf{k}\right\rangle\right|^{2} \delta\left(\varepsilon_{\mathbf{k}}-\hbar \omega_{\mathrm{LO}}\right. \\
& \left.+\varepsilon_{d}\right)
\end{aligned}
$$


where $\varepsilon_{d}$ is the QD level binding energy. $|\mathbf{k}\rangle$ is the initial carrier state in the continuum and $|d\rangle$ is the final carrier state (in the QD). The onset of the continuous energy spectrum is at the QD barrier. We have averaged over the equilibrium phonon distribution in the initial state $|i\rangle$, which gives the factor $(\bar{n}+1)$ with

$$
\bar{n}=\frac{1}{\exp \left(\frac{\hbar \omega_{\mathrm{LO}}}{k_{B} T}\right)-1} .
$$

It is assumed in Eq. (16) that the LO phonons have zero dispersion. The probability, $w(\mathbf{k})$, is now obtained by summing over all emitted phonon modes, $\mathbf{q}$ :

$$
\begin{aligned}
w(\mathbf{k})= & \sum_{\mathbf{q}} w(\mathbf{k}, \mathbf{q}) \\
w(\mathbf{k})= & \frac{2 \pi}{\hbar}(\bar{n}+1) \sum_{\mathbf{q}}\left|C_{q}\right|^{2}\left|\left\langle d\left|e^{-i \mathbf{q} \cdot \mathbf{r}}\right| \mathbf{k}\right\rangle\right|^{2} \delta\left(\varepsilon_{\mathbf{k}}-\hbar \omega_{L O}\right. \\
& \left.+\varepsilon_{d}\right) .
\end{aligned}
$$

Hence,

$$
\begin{aligned}
R= & \sum_{\mathbf{k}} w(\mathbf{k}) f\left(\varepsilon_{\mathbf{k}}\right) \\
= & \frac{2 \pi}{\hbar}(\bar{n}+1) \frac{\hbar \omega_{\mathrm{LO}} e^{2}}{2 \varepsilon_{0} \varepsilon_{r} \Omega} \sum_{\mathbf{k}} \delta\left(\varepsilon_{\mathbf{k}}\right. \\
& \left.-\varepsilon_{k 0}^{1 \mathrm{p}}\right) f\left(\varepsilon_{\mathbf{k}}\right) \sum_{\mathbf{q}} \frac{\left|\left\langle d\left|e^{-i \mathbf{q} \cdot \mathbf{r}}\right| \mathbf{k}\right\rangle\right|^{2}}{q^{2}},
\end{aligned}
$$

where $\varepsilon_{k 0}^{1 \mathrm{p}}=\hbar \omega_{\mathrm{LO}}-\varepsilon_{d}$ and we have inserted $\left|C_{q}\right|^{2}$ from Eq. (13). It is useful to write the capture rate in terms of the 3D density of states. We rewrite Eq. (20) in terms of $g_{3 \mathrm{D}}$, the 3D density of states (DOS),

$$
\begin{aligned}
R= & \frac{2 \pi}{\hbar}(\bar{n}+1) \frac{\hbar \omega_{\mathrm{LO}} e^{2}}{4 \varepsilon_{0} \varepsilon_{r}} \\
& \times g_{3 \mathrm{D}}\left(\varepsilon_{k 0}^{1 \mathrm{p}}\right) f\left(\varepsilon_{k 0}^{1 \mathrm{p}}\right) \frac{1}{4 \pi} \sum_{\mathbf{q}, \mathbf{k}_{0}} \frac{\mid\langle d| e^{-\left.i \mathbf{q} \cdot \mathbf{r}\left|\mathbf{k}_{0}\right\rangle\right|^{2}}}{q^{2}},
\end{aligned}
$$

where $\mathbf{k}_{0}$ fulfills $\varepsilon_{k 0}^{1 \mathrm{p}}=\hbar^{2} k_{0}^{2} /\left(2 m^{*}\right)$ and the summation over $\mathbf{k}_{0}$ denotes an integration over the directions of the vector $\mathbf{k}_{0} \cdot g_{3 \mathrm{D}}$ is given by

$$
g_{3 \mathrm{D}}(\varepsilon)= \begin{cases}\frac{m^{*}}{\pi^{2} \hbar^{2}} \sqrt{\frac{2 m^{*} \varepsilon}{\hbar^{2}}}, & \varepsilon \geqslant 0, \\ 0, & \varepsilon<0 .\end{cases}
$$

Furthermore, a dimensionless "DOS" can be defined in terms of $g_{3 \mathrm{D}}$ :

$$
g_{3 D}(\varepsilon)=\frac{m^{*}}{\pi^{2} \hbar^{2}} \sqrt{\frac{2 m * \hbar \omega_{\mathrm{LO}}}{\hbar^{2}}} d_{3 \mathrm{D}}^{1 \mathrm{p}}(\varepsilon),
$$

where

$$
d_{3 D}^{1 \mathrm{p}}(\varepsilon)= \begin{cases}\sqrt{\frac{\varepsilon}{\hbar \omega_{\mathrm{LO}}}}, & \varepsilon \geqslant 0, \\ 0, & \varepsilon<0 .\end{cases}
$$

$d_{3 \mathrm{D}}^{1 \mathrm{p}}$ has the property that $d_{3 \mathrm{D}}^{1 \mathrm{p}}\left(\varepsilon_{k 0}^{1 \mathrm{p}}\right)=1$ when a new level appears in the QD and $d_{3 \mathrm{D}}^{1 \mathrm{p}}\left(\varepsilon_{k 0}^{1 \mathrm{p}}\right)=0$ when the level becomes too deep for single-phonon capture to be possible. Expression (21) can then be simplified further by writing

$$
R=R_{0}^{1 \mathrm{p}} d_{3 \mathrm{D}}^{1 \mathrm{p}}\left(\varepsilon_{k 0}^{1 \mathrm{p}}\right) f\left(\varepsilon_{k 0}^{1 \mathrm{p}}\right) .
$$

The factor $R_{0}^{1 \mathrm{p}}$ is given by

$$
R_{0}^{1 \mathrm{p}}=\frac{\bar{n}+1}{(4 \pi)^{2} \hbar} \frac{e^{2}}{\varepsilon_{0} \varepsilon_{r}} \kappa^{3} \sum_{\mathbf{q}, \mathbf{k}_{0}} \frac{\left|\left\langle d\left|e^{-i \mathbf{q} \cdot \mathbf{r}}\right| \mathbf{k}_{0}\right\rangle\right|^{2}}{q^{2}},
$$

where $\kappa$ fulfills $\hbar \omega_{\mathrm{LO}}=\hbar^{2} \kappa^{2} /\left(2 m^{*}\right)$. This factor, therefore, gives information about the interaction through the amplitude of the carrier-LO-phonon interaction matrix element and has the unit of $\mathrm{s}^{-1}$ as the capture rate. As described above, the initial state in the barrier can be modeled by a plane wave: ${ }^{5,7,13}$

$$
\langle\mathbf{r} \mid \mathbf{k}\rangle=\frac{1}{\sqrt{\Omega}} e^{i \mathbf{k} \cdot \mathbf{r}} .
$$

This approximation neglects the influence of scattered waves in the QD and hence, in particular, the quasibound states over the dot are not seen.

\section{B. Two-phonon capture}

Capture via emission of two phonons has the advantage that it can take place to states with binding energies $\varepsilon_{d}$ that fulfill $0<\varepsilon_{d} \leqslant 2 \hbar \omega_{\mathrm{LO}}$, whereas single-phonon capture can only take place for $0<\varepsilon_{d} \leqslant \hbar \omega_{\mathrm{LO}}$. For the two-phonon capture, the transition probability can be written as

$$
w(\mathbf{k})=\sum_{\mathbf{q}_{1}, \mathbf{q}_{2}} w\left(\mathbf{k}, \mathbf{q}_{1}, \mathbf{q}_{2}\right),
$$

where $w\left(k, q_{1}, q_{2}\right)$ is the probability of carrier capture via emission of two phonons with wave vectors $\mathbf{q}_{1}$ and $\mathbf{q}_{2}$. Second-order perturbation theory gives ${ }^{20}$ (see the Appendix)

$$
\begin{aligned}
w\left(\mathbf{k}, \mathbf{q}_{1}, \mathbf{q}_{2}\right)= & \frac{2 \pi}{\hbar}(\bar{n}+1)^{2} \\
& \times\left|C_{q_{1}} C_{q_{2}} \sum_{\nu} \frac{M_{f \nu}^{q_{2}} M_{\nu i}^{q_{1}}+M_{f \nu}^{q_{1}} M_{\nu i}^{q_{2}}}{\varepsilon_{\mathbf{k}}-\varepsilon_{\nu}-\hbar \omega_{\mathrm{LO}}}\right|^{2} \delta\left(\varepsilon_{\mathbf{k}}\right. \\
& \left.-2 \hbar \omega_{\mathrm{LO}}+\varepsilon_{d}\right),
\end{aligned}
$$

where the summation is over intermediate carrier states, which either belong to the continuous energy spectrum or the discrete QD spectrum. The matrix elements are given by

$$
\begin{aligned}
& M_{f \nu}^{q}=\left\langle d\left|e^{-i \mathbf{q} \cdot \mathbf{r}}\right| \zeta_{\nu}\right\rangle, \\
& M_{\nu i}^{q}=\left\langle\zeta_{\nu}\left|e^{-i \mathbf{q} \cdot \mathbf{r}}\right| \mathbf{k}\right\rangle,
\end{aligned}
$$

where $\left|\zeta_{\nu}\right\rangle$ denotes an intermediate carrier state and $\varepsilon_{\nu}$ is the energy of the intermediate carrier state. Figure 2 shows the two-phonon capture process, where capture via the different 


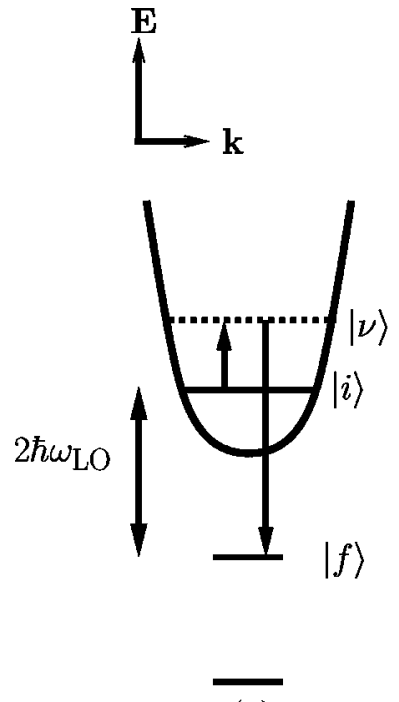

(a)

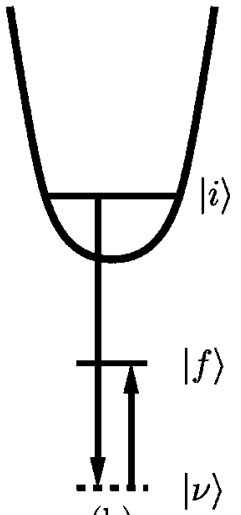

(b)
FIG. 2. Energy diagram in $\mathbf{k}$ space of the two-phonon capture process via different intermediate carrier states $|\nu\rangle$. Dashed lines indicate that a carrier is not actually transferred to an intermediate state. $|i\rangle$ and $|f\rangle$ denote the initial and final state. (a) shows a contribution to the capture from an intermediate continuous state and (b) shows a contribution to the capture from an intermediate discrete state. Initial and final states can also serve as intermediate states. Discrete energy levels do not have any definite $\mathbf{k}$ but are, for illustration purposes, shown here with a finite extension in $\mathbf{k}$ space.

intermediate states is illustrated schematically. Intermediate states are depicted with dashed lines since the carrier is not actually transferred to an intermediate state.

It should be emphasized at this point that the above formula (29) is valid in the case where the denominator in the sum, $\varepsilon_{\mathbf{k}}-\varepsilon_{\nu}-\hbar \omega_{\mathrm{LO}}$, is not too close to zero. The condition $\varepsilon_{\mathbf{k}}-\varepsilon_{\nu}=\hbar \omega_{\mathrm{LO}}$ corresponds, in fact, to a cascaded emission of LO phonons, which cannot be treated perturbatively (see Ref. 27 for a detailed treatment). We restrict ourselves in this article to calculating two-phonon capture rates to states with binding energies $\hbar \omega_{\mathrm{LO}}<\varepsilon_{d} \leqslant 2 \hbar \omega_{\mathrm{LO}}$, where single-phonon capture processes are prohibited. Due to the energy conservation expressed with the $\delta$ function in Eq. (29), $\varepsilon_{\mathbf{k}}$ $=2 \hbar \omega_{\mathrm{LO}}-\varepsilon_{d}$, we have that $0 \leqslant \varepsilon_{\mathbf{k}}<\hbar \omega_{\mathrm{LO}}$ and, in that case, there is not a possibility for a cascaded process via an intermediate carrier state in the continuous energy spectrum. However, a cascaded process can occur via intermediate discrete carrier states, if such a state is situated $\hbar \omega_{\text {LO }}$ below the initial carrier state. Such a situation only takes place for very specific values of confinement potential, dot size, and particle mass and we will avoid this particular situation in this article.

From Eq. (28), we obtain

$$
\begin{aligned}
w(\mathbf{k})= & \frac{2 \pi}{\hbar}(\bar{n}+1)^{2} \\
& \times \sum_{\mathbf{q}_{1}, \mathbf{q}_{2}}\left|C_{q_{1}} C_{q_{2}} \sum_{\nu} \frac{M_{f \nu}^{q_{2}} M_{\nu i}^{q_{1}}+M_{f \nu}^{q_{1}} M_{\nu i}^{q_{2}}}{\varepsilon_{\mathbf{k}}-\varepsilon_{\nu}-\hbar \omega_{\mathrm{LO}}}\right|^{2} \\
& \times \delta\left(\varepsilon_{\mathbf{k}}-2 \hbar \omega_{\mathrm{LO}}+\varepsilon_{d}\right) .
\end{aligned}
$$

Using Eq. (8) we now obtain for the capture rate

$$
\begin{aligned}
& R=\frac{2 \pi}{\hbar}(\bar{n}+1)^{2}\left(\frac{\hbar \omega_{\mathrm{LO}} e^{2}}{2 \varepsilon_{0} \varepsilon_{r}}\right)^{2} \frac{1}{\Omega^{2}} \\
& \times \sum_{\mathbf{k}} f\left(\varepsilon_{\mathbf{k}}\right) \delta\left(\varepsilon_{\mathbf{k}}\right. \\
& \left.-\varepsilon_{k 0}^{2 \mathrm{p}}\right) \sum_{\mathbf{q}_{1}, \mathbf{q}_{2}} \frac{1}{q_{1}^{2} q_{2}^{2}}\left|\sum_{\nu} \frac{M_{f \nu}^{q_{2}} M_{\nu i}^{q_{1}}+M_{f \nu}^{q_{1}} M_{\nu i}^{q_{2}}}{\varepsilon_{\mathbf{k}}-\varepsilon_{\nu}-\hbar \omega_{\mathrm{LO}}}\right|^{2},
\end{aligned}
$$

where $\varepsilon_{k 0}^{2 \mathrm{p}}=2 \hbar \omega_{\mathrm{LO}}-\varepsilon_{d}$ and we have inserted $\left|C_{q}\right|^{2}$ from Eq. (13). This expression can be rewritten in terms of the 3D DOS in the same manner as Eq. (21) was obtained

$$
\begin{aligned}
R= & \frac{2 \pi}{\hbar}(\bar{n}+1)^{2}\left(\frac{\hbar \omega_{\mathrm{LO}} e^{2}}{4 \varepsilon_{0} \varepsilon_{r}}\right)^{2} \\
& \times g_{3 \mathrm{D}}\left(\varepsilon_{k 0}^{2 \mathrm{p}}\right) f\left(\varepsilon_{k 0}^{2 \mathrm{p}}\right) \frac{1}{2 \pi \Omega} \\
& \times \sum_{\mathbf{q}_{1}, \mathbf{q}_{2}, \mathbf{k}_{0}} \frac{1}{q_{1}^{2} q_{2}^{2}}\left|\sum_{\nu} \frac{M_{f \nu}^{q_{2}} M_{\nu i}^{q_{1}}+M_{f \nu}^{q_{1}} M_{\nu i}^{q_{2}}}{\varepsilon_{k 0}^{2 \mathrm{p}}-\varepsilon_{\nu}-\hbar \omega_{\mathrm{LO}}}\right|^{2},
\end{aligned}
$$

where the sum over $\mathbf{k}_{0}$ denotes, as before, an integral over the directions of $\mathbf{k}_{0}$. This expression can also be written in terms of a dimensionless DOS

$$
R=R_{0}^{2 \mathrm{p}} d_{3 \mathrm{D}}^{2 \mathrm{p}}\left(\varepsilon_{k 0}^{2 \mathrm{p}}\right) f\left(\varepsilon_{k 0}^{2 \mathrm{p}}\right),
$$

where

$$
\begin{aligned}
R_{0}^{2 \mathrm{p}}= & \frac{(\bar{n}+1)^{2}}{\sqrt{2}(4 \pi)^{2} \hbar}\left(\frac{e^{2}}{\varepsilon_{0} \varepsilon_{r}}\right)^{2} \\
& \times \kappa^{3} \hbar \omega_{\mathrm{LO}} \frac{1}{\Omega} \sum_{\mathbf{q}_{1}, \mathbf{q}_{2}, \mathbf{k}_{0}} \frac{1}{q_{1}^{2} q_{2}^{2}} \\
& \times\left|\sum_{\nu} \frac{M_{f \nu}^{q_{2}} M_{\nu i}^{q_{1}}+M_{f \nu}^{q_{1}} M_{\nu i}^{q_{2}}}{\varepsilon_{k 0}^{2 \mathrm{p}}-\varepsilon_{\nu}-\hbar \omega_{\mathrm{LO}}}\right|^{2} .
\end{aligned}
$$

The dimensionless DOS for this two-phonon capture process is defined as

$$
d_{3 \mathrm{D}}^{2 \mathrm{p}}(\varepsilon)= \begin{cases}\sqrt{\frac{\varepsilon}{2 \hbar \omega_{\mathrm{LO}}},}, & \varepsilon \geqslant 0, \\ 0, & \varepsilon<0,\end{cases}
$$

which is analogous to the definition of $d_{3 \mathrm{D}}^{1 \mathrm{p}}$ in Eq. (24). $d_{3 \mathrm{D}}^{2 \mathrm{p}}\left(\varepsilon_{k 0}^{2 \mathrm{p}}\right)$ is equal to unity when a new level appears in the QD and becomes zero when the level becomes too deep for two-phonon capture to be possible.

For capture via intermediate continuum states, we have the selection rule

$$
M_{\nu i}^{q}=\delta_{\mathbf{k}, \mathbf{k}_{\nu}+\mathbf{q}},
$$

since the incident carrier wave function is described by a plane wave (27). This implies crystal momentum conservation in the continuum. With this selection rule, the matrix elements in the sum involving continuum intermediate states are given by 


$$
\begin{aligned}
& M_{f \nu}^{q_{2}} M_{\nu i}^{q_{1}}=F\left(\mathbf{k}-\mathbf{q}_{1}-\mathbf{q}_{2}\right), \\
& M_{f \nu}^{q_{1}} M_{\nu i}^{q_{2}}=M_{f \nu}^{q_{2}} M_{\nu i}^{q_{1}},
\end{aligned}
$$

where $F\left(\mathbf{k}-\mathbf{q}_{1}-\mathbf{q}_{2}\right) \equiv 1 / \sqrt{\Omega} \int d \mathbf{r} \Psi_{d}^{*}(\mathbf{r}) e^{i\left(\mathbf{k}-\mathbf{q}_{1}-\mathbf{q}_{2}\right) \cdot \mathbf{r}}$. With this definition, $R_{0}^{2 \mathrm{p}}$ becomes

$$
\begin{aligned}
& R_{0}^{2 \mathrm{p}}=\frac{(\bar{n}+1)^{2}}{\sqrt{2}(4 \pi)^{2} \hbar}\left(\frac{e^{2}}{\varepsilon_{0} \varepsilon_{r}}\right)^{2} \kappa^{3} \hbar \omega_{\mathrm{LO}} \frac{1}{\Omega} \sum_{\mathbf{q}_{1}, \mathbf{q}_{2}, \mathbf{k}_{0}} \frac{1}{\mathbf{q}_{1}^{2} \mathbf{q}_{2}^{2}} \\
& \times \mid \frac{F\left(\mathbf{k}_{0}-\mathbf{q}_{1}-\mathbf{q}_{2}\right)}{\varepsilon_{\mathbf{k}_{0}}-\varepsilon_{\mathbf{k}_{0}-\mathbf{q}_{1}}-\hbar \omega_{\mathrm{LO}}}+\frac{F\left(\mathbf{k}_{0}-\mathbf{q}_{1}-\mathbf{q}_{2}\right)}{\varepsilon_{\mathbf{k}_{0}}-\varepsilon_{\mathbf{k}_{0}-\mathbf{q}_{2}}-\hbar \omega_{\mathrm{LO}}} \\
& +\left.\sum_{p_{\nu}} \frac{M_{f \nu}^{q_{2}} M_{\nu i}^{q_{1}}+M_{f \nu}^{q_{1}} M_{\nu i}^{q_{2}}}{\varepsilon_{k 0}^{2 \mathrm{p}}-\varepsilon_{\nu}-\hbar \omega_{\mathrm{LO}}}\right|^{2},
\end{aligned}
$$

where $p_{\nu}$ stands for the quantum numbers describing intermediate discrete QD states.

\section{Quantum-dot wave functions and energy levels}

In a real situation of self-assembled QDs, the dots are formed on the interface of two lattice-mismatched materials. The dots' shape is in many cases believed to be a truncated pyramid, ${ }^{28}$ coupled to a common two-dimensional layer; the wetting layer. Carriers are captured into the dots primarily via this $2 \mathrm{D}$ layer. Moreover, the QD energy spectra and wave functions are strongly modified due to the presence of strain in these structures ${ }^{29,33}$ and, in fact, the precise size and shape of dots is not known. The purpose of this article is to investigate two-phonon capture processes and the time scale on which these occur in comparison to single-phonon processes. To achieve this purpose, we use the spherical model of a QD with finite confinement

$$
V(\mathbf{r})= \begin{cases}0, & r \leqslant a, \\ V_{0} & r>a,\end{cases}
$$

where $V_{0}>0$ and $a$ is the QD radius. In this case, the wave function for bound QD states is given by

$$
\Psi_{d}(\mathbf{r})=Y_{\ell}^{m}(\theta, \phi) G_{\ell n}(r),
$$

where $Y^{m}$ are the spherical harmonics and the radial part of the wave function, $G_{\ell n}$, can be expressed through the spherical Bessel function (see, for instance, Ref. 30). The number $n$ stands for the $n$th level of angular momentum $\ell$, which becomes bound to the dot. The quantum number $n$ should not be confused with the carrier density. Note that the spherical model is explored effectively in modeling of chemically synthesized colloidal QDs, which have a spherical shape, and on which gain has recently been obtained. ${ }^{31,32}$

\section{RESULTS}

The material parameters are chosen to be those of bulk GaAs. The phonon energy is set to be $\hbar \omega_{\mathrm{LO}}=35 \mathrm{meV}$. The carriers are assumed to be electrons of effective mass $m^{*}$ $=0.07 m_{0}$, where $m_{0}$ is the free-electron mass. The confinement potential is set to be $V_{0}=200 \mathrm{meV}$ (close to the value in Ref. 33). Furthermore, unless otherwise stated, we use $T$ $=300 \mathrm{~K}$ and $n=10^{17} \mathrm{~cm}^{-3}$. Figure 3 shows the calculated

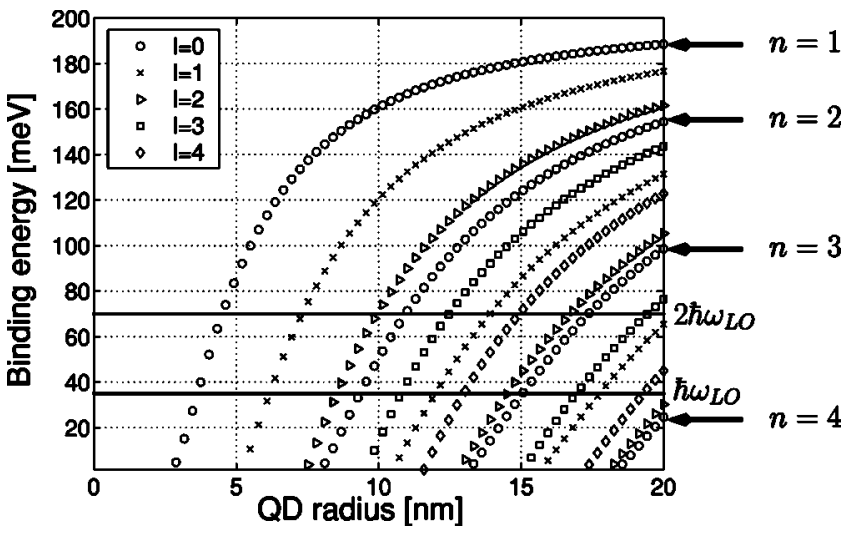

FIG. 3. Binding energy of an electron in a spherically symmetric and finite potential as a function of the QD radius. Results are shown for angular momenta $\ell \in[0,4]$. The horizontal lines indicate $\hbar \omega_{\text {LO }}$ (LO phonon energy) and $2 \hbar \omega_{\mathrm{LO}}$. A carrier can be captured into states with binding energies below $\hbar \omega_{\mathrm{LO}}$ in the case of single-phonon capture and below $2 \hbar \omega_{\mathrm{LO}}$ in the two-phonon capture case. Quantum number $n$ is shown for $\ell=0$ for illustration. For instance, $n=1$ is the first $\ell=0$ state to become bound, $n$ $=2$ the second, etc.

binding energy as a function of the QD radius. Up to $\approx 2.8 \mathrm{~nm}$, there is no bound state in the dot. This is in contrast to the case of quantum wells (QWs), where at least one state is bound. Due to the spherical symmetry, QD states can be characterized by an angular momentum quantum number $\ell$, an azimuthal quantum number $m$, and spin $s$. Each level $\ell$ is $2(2 \ell+1)$ degenerate. Instead of determining the capture rate, $R_{\ell, m}^{s}$, into each state $(\ell, m, s)$, we define an effective capture rate

$$
R_{\ell}=2 \sum_{m=-\ell}^{\ell} R_{\ell, m}^{s},
$$

for which $\tau_{\ell}=1 / R /$ is the photoluminescence rise time of a given level $\ell$. The factor of 2 arises from spin degeneracy of the QD level. It should be emphasized that rate equation (1) describes occupation probability $\rho$ of a given state and expression (42) cannot be inserted into rate equations of the form in Eqs. (1) and (2). If $\rho_{\ell, m}^{s}$ is the occupation probability of a state $(\ell, m, s)$, we have

$$
\frac{\partial \rho_{\ell, m}^{s}}{\partial t}=R_{\ell, m}^{s}\left(1-\rho_{\ell, m}^{s}\right),
$$

and the rate equation for $n$ includes contributions from all states $(\ell, m, s)$;

$$
\frac{\partial n}{\partial t}=-2 N_{D} \sum_{\ell, m} R_{\ell, m}\left(1-\rho_{\ell m}\right) .
$$

In Eq. (44), we have used that $R_{\ell, m}^{+1 / 2}=R_{\ell, m}^{-1 / 2} \equiv R_{\ell, m}$, and hence, the factor of 2 .

\section{A. Single-phonon capture}

The single-phonon capture time for the individual angular momentum states, $\ell$, i.e., $\tau_{\ell}$, is shown in Fig. 4 as a function of QD radius. Capture to states of all $\ell$ within $\hbar \omega_{\mathrm{LO}}$ from the QD barrier is possible; results for $\ell$ $\in[0,4]$ are shown. It is seen that there are bands of dot radii where phonon-mediated capture is allowed, consistent with 


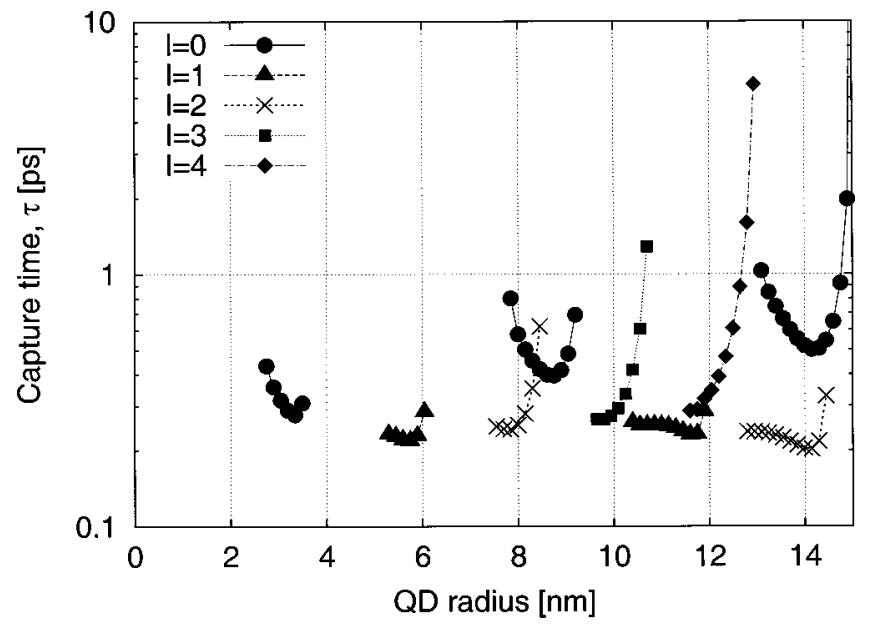

FIG. 4. Single-phonon capture time $\tau_{\ell}$ as a function of QD radius for angular momenta $\ell \in[0,4] . T=300 \mathrm{~K}$ and $n=10^{17} \mathrm{~cm}^{-3}$.

other results. ${ }^{7}$ The bands are seen to nearly cover the dot size range apart from two narrow gaps on the lower radius side. The lower end of a radius band, $a=a_{\min }$, is defined by the appearance of a new level in the QD to which capture may take place. The increase in $\tau$ at this end essentially follows the decrease in $R_{0}^{1 \mathrm{p}}$ as $a \rightarrow a_{\mathrm{min}}^{+}$. In this limit, the "volume" of the QD wave function tends to infinity due to the weaker binding to the dot and $R_{0}^{1 \mathrm{p}}$ decreases. The rate approaches zero at the higher end of each radius band ( $\tau$ approaches infinity) beyond which the binding energy of the level is larger than the phonon energy. The abrupt increase in $\tau$ at this end can be explained in terms of the DOS of the incident carrier as $\varepsilon_{k 0}^{1 \mathrm{p}} \rightarrow 0^{+}$[see Eqs. (24) and (25)]. The capture times are typically of the order of $0.2-0.3 \mathrm{ps}$ at the radius band minima. In general, our results obtained with the spherical QD model are close to the results of Ref. 7 for a cone QD.

We have also considered the dependence of the capture times on confinement energy. We decreased the confinement energy by a factor of $2 ; V_{0}=100 \mathrm{meV}$, and calculated the capture times for the first $\ell=0$ band. The capture time minimum is found to be similar to the case of $V_{0}=200 \mathrm{meV}$ but

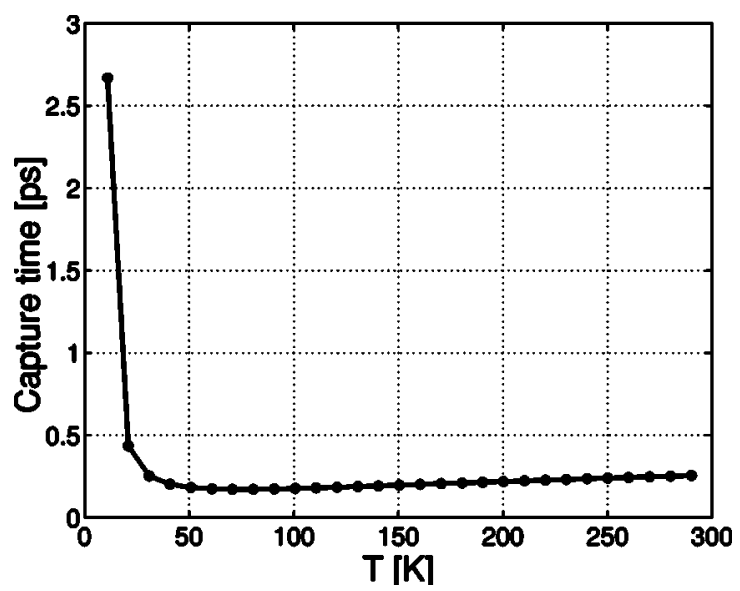

FIG. 5. Temperature dependence of single-phonon capture time $\tau_{\ell}$ into the second $\ell=0$ band, at $a=8.7 \mathrm{~nm} . n=10^{17} \mathrm{~cm}^{-3}$.

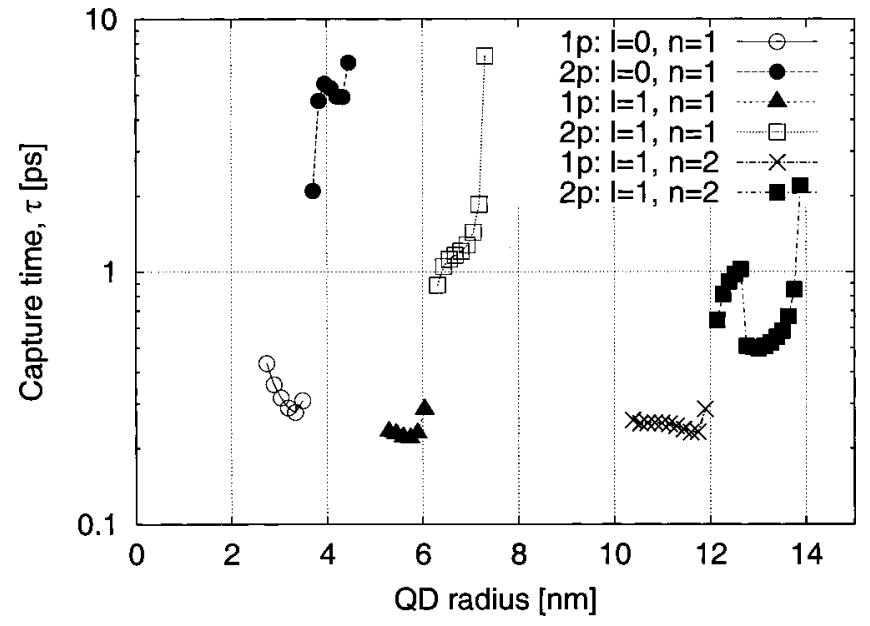

FIG. 6. Two-phonon capture time $\tau_{\ell}$ (labeled " $2 \mathrm{p}$ ") as a function of QD radius into the QD levels $\ell=0, n=1$ and $\ell=1$ with $n=1,2$. Singlephonon capture times (labeled "1p") into the same states are shown for comparison. Two-phonon capture into $\ell=0, n=2,3$ cannot be shown, since the perturbative approach breaks down when the separation of energy levels is too close to $\hbar \omega_{\mathrm{LO}}$ (see Fig. 3). $T=300 \mathrm{~K}$ and $n=10^{17} \mathrm{~cm}^{-3}$.

the radius band is broader, $2.5 \mathrm{~nm}$ wide, while it is less than $1 \mathrm{~nm}$ wide for $V_{0}=200 \mathrm{meV}$. The $\tau$ dependence on QD radius is thus less pronounced.

The dependence of the capture rate on temperature for fixed carrier density is shown in Fig. 5. The temperature dependence occurs via the phonon and Fermi distribution functions as

$$
R_{T} \sim f\left(\varepsilon_{k 0}^{1 \mathrm{p}}\right)(\bar{n}+1) .
$$

The behavior of the capture time with temperature follows mainly the behavior of the Fermi factor, since the phonon population factor varies slowly. At low temperatures, both $f\left(\varepsilon_{k 0}^{1 \mathrm{p}}\right)$ and $\bar{n}$ are nearly zero, giving infinite capture times. At $T \approx 50 \mathrm{~K}$, the capture time reaches a minimum. At low and moderate carrier densities, the minimum occurs at the same temperature. The capture time stays approximately constant ( $\tau \approx 0.3 \mathrm{ps}$ ) for temperatures above $\sim 50 \mathrm{~K}$.

\section{B. Two-phonon capture}

In Fig. 6 we show the two-phonon capture time of an electron, $\tau_{\ell}$, along with the single-phonon capture time from Fig. 4 for comparison. In general, the radius bands for twophonon capture processes are more than two times as large as the corresponding band for single-phonon capture as the slope of the binding energy (Fig. 3) decreases with increasing radius. Here, we restrict ourselves to capture into states that have binding energy $\hbar \omega_{\mathrm{LO}}<\varepsilon_{d} \leqslant 2 \hbar \omega_{\mathrm{LO}}$ and for which the perturbative approach is valid. One can see that the calculated two-phonon capture times into $\ell=0, n=1$ and $\ell$ $=1, n=1$ levels are of the order of some picoseconds at $n$ $=10^{17} \mathrm{~cm}^{-3}$. This is approximately an order of magnitude longer than the single-phonon capture times into these states. The two-phonon capture time into the $\ell=1, n=2$ level is close to $1 \mathrm{ps}$, which is only slightly longer than the singlephonon capture time.

We have investigated the influence of the different intermediate states on the total carrier capture rate by "turning off" contributions from other states. This analysis has been 
performed for the the first radius band, $\ell=0, n=1$, where a single energy level is present in the dot. For instance, at a binding energy $\varepsilon_{d} \approx 2 \hbar \omega_{\mathrm{LO}}$, the contribution from continuum intermediate states (neglecting spin degeneracy) gives a capture rate $R=2 \times 10^{11} \mathrm{~s}^{-1}(\tau=5 \mathrm{ps})$ and the corresponding contribution from the state itself gives $R=4$ $\times 10^{11} \mathrm{~s}^{-1}$. However, the total capture rate, with all contributions included, is smaller; $R=8 \times 10^{10} \mathrm{~s}^{-1}(\tau=12.5 \mathrm{ps})$. This suggests that destructive interference between the two contributions takes place. Mathematically, this can be explained by the difference in sign of the denominator in Eq. (29) corresponding to the different contributions. The denominator corresponding to continuous intermediate states is always negative. Denominators for discrete intermediate states can be either positive or negative depending on the relative level position, the crossing point from negative to positive occuring when an intermediate (discrete) state is $\hbar \omega_{\text {LO }}$ above the state to be captured into. The interference pattern becomes more complex as more states become bound to the dot and is expected to depend strongly on the energy separation between the different dot levels.

The case of capture into the $\ell=0, n=2,3$ band cannot be shown since, in this case, there exist discrete intermediate carrier states lying $\hbar \omega_{\text {LO }}$ above the state to be captured into (see Fig. 3). In this case the perturbative approach that we have chosen here is no longer valid (see discussion in Sec. II B).

In the case of capture into an $l=1, n=1$ state, two states are bound to the dot, an $l=0$ state lying deep within the dot and the level $l=1$. Performing an analysis of the contribution from different intermediate states in this situation shows that although the matrix elements corresponding to different intermediate states are approximately of the same order, the contribution via continuous states is the most dominant. This is explained in terms of the denominators; the ones corresponding to the discrete intermediate states are equal to or larger than $\hbar \omega_{\mathrm{LO}}$ while the denominator for continuous states is small and negative. In this particular case, interference between continuous contributions on one hand, discrete on the other, is negligible as the calculations show that they are $\pi / 2$ out of phase.

In the $\ell=1, n=2$ band, a local decrease is seen in the capture time at $a=12.8 \mathrm{~nm}$, found to be associated with the binding of the $\ell=2, n=2$ state. However, no decrease is observed in the capture time with the binding of $\ell=0, n$ $=3$ at $a=13 \mathrm{~nm}$. This can be understood in terms of overlap of the different radial wave functions with the final state wave function. The radial wave functions are shown in Fig. 7 at $a=13.1 \mathrm{~nm}$. $G_{12}$ and $G_{22}$ have the same number of nodes and behave very similarly. Hence, their overlap is very good, resulting in the local increase in the capture rate. $G_{03}$ is out of phase with those functions and, therefore, does not contribute significantly to the capture rate. No step is observed in the capture rate into $\ell=1, n=1$ as no new state becomes bound in the dot in this band. We have limited the number of intermediate states to those with $\ell \in[0,5]$. As the first $\ell$ $=6$ state becomes bound at $a=15.4 \mathrm{~nm}$, we only show results up to this QD radius.

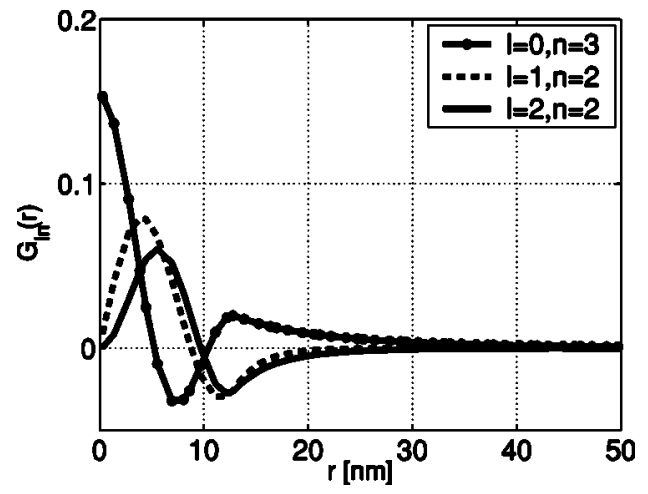

FIG. 7. Radial wave functions at $a=13.1 \mathrm{~nm}$.

The discussion above shows clearly that the total capture rate depends strongly on the interference between contributions from the different intermediate states. The character of the interference can to a large extent be identified by the sign of the denominators of the different terms that enter into the capture rate [see Eqs. (29), (33), and (39)]. Therefore, an increase of possible intermediate states does not necessarily lead to a simple increase in the capture rate. Furthermore, the contribution of an intermediate state depends strongly on the overlap of its radial wave function with the radial wave function of the final state. This was shown in the case of carrier capture into the $\ell=1, n=2$ level.

The capture time dependence on carrier density is shown in Fig. 8 for single- and two-phonon capture into the first $\ell=0$ band (see Fig. 6). The density dependence occurs via the Fermi filling of the energy level of the incident carrier. At low and moderate carrier densities, the Fermi distribution approaches a Boltzmann distribution,

$$
f_{B}\left(\varepsilon_{\mathbf{k}}\right)=\frac{n}{\bar{n}_{3 \mathrm{D}}} \exp \left(-\frac{\varepsilon_{\mathbf{k}}}{k_{B} T}\right),
$$

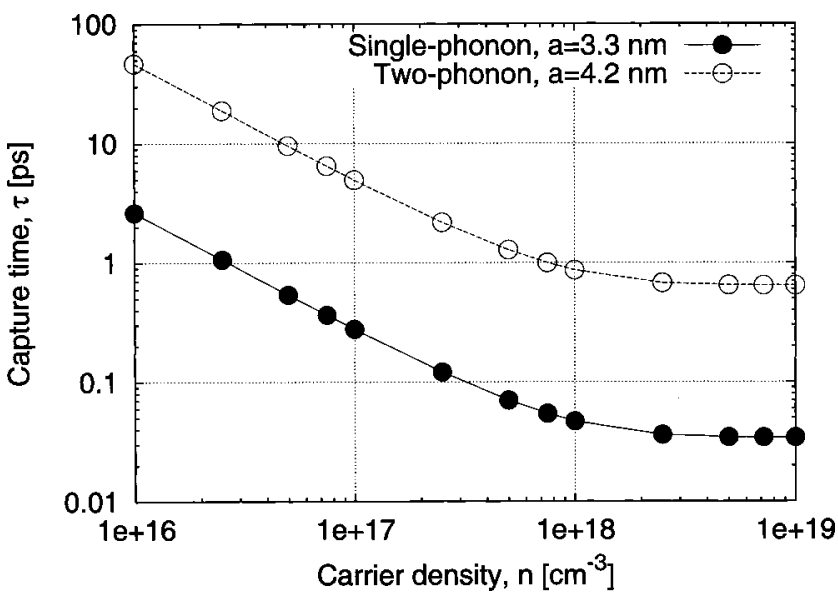

FIG. 8. Density dependence of single- and two-phonon capture times $\tau_{\ell}$ for two different dot sizes in the first $\ell=0$ band. At $a=3.3 \mathrm{~nm}$, only the singlephonon capture can be calculated with the perturbative formula for the capture rate. Only the two-phonon process is allowed at $a=4.2 \mathrm{~nm}$. A coefficients, defined by Eq. (47), are found to be $A^{1 \mathrm{p}}=3.8 \times 10^{-5} \mathrm{~cm}^{3} / \mathrm{s}$ for the single-phonon capture and for the two-phonon capture $A^{2 \mathrm{p}}=1.9$ $\times 10^{-6} \mathrm{~cm}^{3} / \mathrm{s}$, i.e., one order of magnitude smaller. 
$\bar{n}_{3 \mathrm{D}}=2\left[m^{*} k_{B} T /\left(2 \pi \hbar^{2}\right)\right]^{3 / 2}$ being the 3D density of states. At these densities we can, therefore, define

$$
R=A n,
$$

as was already stated in Sec. II. The coefficients $A$ are extracted from Fig. 8. The value for the single-phonon process is $A^{1 \mathrm{p}}=3.8 \times 10^{-5} \mathrm{~cm}^{3} \mathrm{~s}^{-1}$, and $A^{2 \mathrm{p}}=1.9 \times 10^{-6} \mathrm{~cm}^{3} \mathrm{~s}^{-1}$ for the two-phonon capture. The effective capture time can now be evaluated from Eq. (7) using the calculated values for the $A$ coefficients once dot density $N_{D}$ has been estimated. In QD-based devices, several layers of dots are grown to increase their gain. To estimate a typical value of $N_{D}$, we assume that the interlayer distance is about $20 \mathrm{~nm}$. For a typical areal dot density $5 \times 10^{14} \mathrm{~m}^{-2}$, the dot density is then $N_{D}=2.5 \times 10^{16} \mathrm{~cm}^{-3}$, and the effective capture times for single- and two-phonon processes become $\tau_{c}^{1 p}=1.1 \mathrm{ps}$ and $\tau_{c}^{2 p}=21$ ps. It should, however, be emphasized that the $A$ values depend strongly on the QD radius.

Alternatively, the capture rate can be expressed through the capture cross section as

$$
R=\sigma n v_{T},
$$

where $v_{T}$ is the thermal velocity of the electron gas. By comparing Eq. (48) with Eq. (47), we obtain that $\sigma^{1 \mathrm{p}}=1.5$ $\times 10^{-12} \mathrm{~cm}^{2}$ and $\sigma^{2 \mathrm{p}}=7.5 \times 10^{-14} \mathrm{~cm}^{2}$. The latter value is close to an estimate in a calculation of gain dynamics in QD lasers. ${ }^{34}$

At very high carrier densities, the capture rate saturates due to the saturation in the Fermi filling factors. As seen from Fig. 8, this occurs at nearly the same carrier density for the two different processes. This arises from the fact that the captured carriers have nearly the same energy $\left(\varepsilon_{k 0}^{1 \mathrm{p}}\right.$ $=12.8 \mathrm{meV}$ and $\varepsilon_{k 0}^{2 \mathrm{p}}=11.4 \mathrm{meV}$ ) and the Fermi filling behaves, therefore, in a similar fashion.

\section{CONCLUSIONS}

In conclusion, we have calculated capture times in two LO-phonon-mediated capture processes. The dependence on dot radius, angular momentum quantum number, and carrier density has been investigated. The case of single-phononmediated capture has also been analyzed and the radius bands, where single-phonon capture is allowed, were identified. For a typical carrier density, $n=10^{17} \mathrm{~cm}^{-3}$, the minima in single-phonon capture times were typically found to lie between 0.2 and $0.3 \mathrm{ps}$. In general, the radius bands in the two-phonon capture case were found to be more than two times as large as single-phonon radius bands. We have calculated the two-phonon capture times in the regions where single-phonon capture is not possible. The role of both continuous and discrete intermediate carrier states in twophonon capture was investigated. The importance of the different intermediate states was shown to be dependent upon the relative energy position of the different dot levels and an increase of possible intermediate states does not lead to a simple increase in the capture rate. The influence of the different discrete intermediate states on the total capture rate was shown to depend strongly on the overlap of the radial wave functions of the intermediate and final states. It was found that the two-phonon capture times into $\ell=0, n=1$ and $\ell=1, n=1$ levels are of the order of some picoseconds at $n=10^{17} \mathrm{~cm}^{-3}$. This is an order of magnitude longer than the single-phonon capture times into these states. The twophonon capture time into $\ell=1, n=2$ is about 1 ps (at $n$ $=10^{17} \mathrm{~cm}^{-3}$ ), which is slightly longer than the singlephonon capture time into this level. The two-phononmediated capture has thus been found to contribute efficiently in situations where single-phonon capture is energetically not allowed.

\section{ACKNOWLEDGMENTS}

The work of one of the authors (A.V.U.) was supported by the Russian Federal Program Integration (Project No. A0155), by RFBR (Project No. 01-02-17330), by INTAS (Project No. 2001-0571), by the Danish Research Council within the framework of program SCOOP, and by the Otto Moensted Foundation.

\section{APPENDIX: DERIVATION OF THE TRANSITION PROBABILITY OF CAPTURE VIA EMISSION OF TWO LONGITUDINAL OPTICAL PHONONS}

The purpose of this Appendix is to give the details in the derivation of Eq. (29), i.e., the transition probability that a carrier with wave vector $\mathbf{k}$ is captured by emitting phonons with given wave vectors $\mathbf{q}_{1}$ and $\mathbf{q}_{2}$. The probability is given by second-order perturbation theory: ${ }^{20}$

$$
w\left(\mathbf{k}, \mathbf{q}_{1}, \mathbf{q}_{2}\right)=\frac{2 \pi}{\hbar}\left|\sum_{\nu} \frac{V_{\mathrm{f} \nu}^{\mathrm{em}} V_{\nu \mathrm{i}}^{\mathrm{em}}}{E_{i}-E_{\nu}}\right|^{2} \delta\left(\varepsilon_{\mathbf{k}}-2 \hbar \omega_{\mathrm{LO}}+\varepsilon_{d}\right),
$$

where the states, for which $V_{f \nu}$ and $V_{\nu i}$ are different from zero, are referred to as intermediate states. The Hamiltonian for emission of two phonons is given by

$$
\hat{V}^{\mathrm{em}}\left(\mathbf{q}_{1}, \mathbf{q}_{2}\right)=C_{q_{1}}^{*} e^{-i \mathbf{q}_{1} \cdot \mathbf{r}} \hat{a}_{\mathbf{q}_{1}}^{\dagger}+C_{q_{2}}^{*} e^{-i \mathbf{q}_{2} \cdot \mathbf{r}} \hat{a}_{\mathbf{q}_{2}}^{\dagger},
$$

and $E_{i}\left(E_{\nu}\right)$ is the energy of the initial (intermediate) state. In this two-phonon capture process, the carrier relaxes through an intermediate state, $|\nu\rangle$, where its electronic part is either a state in the continuous part of the energy spectrum $\left|\mathbf{k}_{\nu}\right\rangle$, or a discrete QD state, $\left|d_{\nu}\right\rangle$. Hereafter, intermediate carrier states are labeled $\left|\zeta_{\nu}\right\rangle$. The states involved in the process are labeled as follows:

$$
\begin{aligned}
& |i\rangle=|\mathbf{k}\rangle\left|\left\{n_{\mathbf{q}}^{i}\right\}\right\rangle, \\
& |f\rangle=|d\rangle\left|\left\{n_{\mathbf{q}}^{f}\right\}\right\rangle, \\
& |\nu\rangle=\left|\zeta_{\nu}\right\rangle\left|\left\{n_{\mathbf{q}}^{\nu}\right\}\right\rangle .
\end{aligned}
$$

We assume that the phonons are emitted into two different phonon modes, which we label $\mathbf{q}_{1}$ and $\mathbf{q}_{2}$, so that the initial and final phonon states differ only in the number of phonons in modes $\mathbf{q}_{1}$ and $\mathbf{q}_{2}$,

$$
\begin{aligned}
& n_{\mathbf{q}_{1}}^{f}=n_{\mathbf{q}_{1}}^{i}+1, \\
& n_{\mathbf{q}_{2}}^{f}=n_{\mathbf{q}_{2}}^{i}+1 .
\end{aligned}
$$


It can be shown that the contribution from phonons emitted into the same mode is negligible. If we assume that the wave vector of the first emitted phonon is $\mathbf{q}_{1}$, so that

$$
n_{\mathbf{q}_{1}}^{\nu}=n_{\mathbf{q}_{1}}^{i}+1,
$$

the transition matrix element from $|i\rangle$ to $|\nu\rangle$ is given by

$$
\begin{aligned}
& V_{\nu i}^{\mathrm{em}}=\left\langle\left\{n_{\mathbf{q}}^{\nu}\right\}\left|\left\langle\zeta_{\nu}\left|\hat{V}^{\mathrm{em}}\right| \mathbf{k}\right\rangle\right|\left\{n_{\mathbf{q}}^{i}\right\}\right\rangle,
\end{aligned}
$$

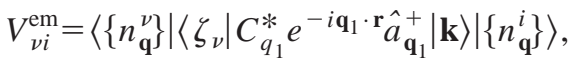

$$
\begin{aligned}
& V_{\nu i}^{\mathrm{em}}=\sqrt{n_{q_{1}}^{i}+1} C_{q_{1}}^{*}\left\langle\zeta_{\nu}\left|e^{-i \mathbf{q}_{1} \cdot \mathbf{r}}\right| \mathbf{k}\right\rangle, \\
& V_{\nu i}^{\mathrm{em}}=\sqrt{n_{q_{1}}^{i}+1} C_{q_{1}}^{*} M_{\nu i}^{q_{1}} .
\end{aligned}
$$

Correspondingly, the transition matrix element from $|\nu\rangle$ to $|f\rangle$ is given by

$$
\begin{aligned}
& V_{f \nu}=\sqrt{n_{q_{2}}^{i}+1} C_{q_{2}}^{*}\left\langle d\left|e^{-i \mathbf{q}_{2} \cdot \mathbf{r}}\right| \zeta_{\nu}\right\rangle, \\
& V_{f \nu}=\sqrt{n_{q_{2}}^{i}+1} C_{q_{2}}^{*} M_{f \nu}^{q_{2}} .
\end{aligned}
$$

It is, of course, also possible to label the phonon vectors in the reverse order, such that the sum over intermediate states consists of two contributions. The transition probability then becomes

$$
\begin{aligned}
w_{\text {if }}\left(\mathbf{k}, \mathbf{q}_{1}, \mathbf{q}_{2}\right)= & \frac{2 \pi}{\hbar}(\bar{n}+1)^{2} \\
& \times\left|\sum_{\nu} C_{q_{1}}^{*} C_{q_{2}}^{*} \frac{M_{f \nu}^{q_{2}} M_{\nu i}^{q_{1}}+M_{f \nu}^{q_{1}} M_{\nu i}^{q_{2}}}{\varepsilon_{\mathbf{k}}-\varepsilon_{\nu}-\hbar \omega_{\mathrm{LO}}}\right|^{2} \\
& \times \delta\left(\varepsilon_{k}-2 \hbar \omega_{\mathrm{LO}}+\varepsilon_{d}\right),
\end{aligned}
$$

where $\varepsilon_{\mathbf{k}}\left(\varepsilon_{\nu}\right)$ is the energy of the initial (intermediate) carrier state. It should be noted here that a discrete QD intermediate carrier state can be the same as the final carrier state, since the matrix element $M_{f f}^{q_{i}}, i=1,2$, is in that case generally nonzero. Here, we have replaced $\left(n_{q_{1}}^{i}+1\right)\left(n_{q_{2}}^{i}+1\right)$ with the average $(\bar{n}+1)^{2}$.

${ }^{1}$ D. Bimberg, M. Grundmann, and N. N. Ledentsov, Quantum Dot Heterostructures (Wiley, New York, 1999).

${ }^{2}$ H. Shoji, Y. Nakata, K. Mukai, Y. Sugiyama, M. Sugawara, N. Yokoyama, and H. Ishikawa, IEEE J. Sel. Top. Quantum Electron. 3, 188 (1997).

${ }^{3}$ G. Park, O. B. Shchekin, D. L. Huffaker, and D. G. Deppe, Electron. Lett. 36, 1283 (2000)

${ }^{4}$ S. Raymond, K. Hinzer, S. Fafard, and J. L. Merz, Phys. Rev. B 61, R16331 (2000).

${ }^{5}$ U. Bockelmann and T. Egeler, Phys. Rev. B 46, 15574 (1992).

${ }^{6}$ I. Vurgaftman and J. Singh, Appl. Phys. Lett. 64, 232 (1994).

${ }^{7}$ R. Ferreira and G. Bastard, Appl. Phys. Lett. 74, 2818 (1999).

${ }^{8}$ O. Verzelen, R. Ferreira, and G. Bastard, Phys. Rev. B 62, R4809 (2000).

${ }^{9}$ U. Bockelmann and G. Bastard, Phys. Rev. B 42, 8947 (1990).

${ }^{10}$ T. Inoshita and H. Sakaki, Phys. Rev. B 46, 7260 (1992).

${ }^{11}$ P. A. Knipp and T. L. Reinecke, Phys. Rev. B 52, 5923 (1995).

${ }^{12}$ J. Feldmann, S. T. Cundiff, M. Arzberger, G. Böhm, and G. Abstreiter, J. Appl. Phys. 89, 1180 (2001).

${ }^{13}$ A. V. Uskov, J. McInerney, F. Adler, and H. Schweizer, Appl. Phys. Lett. 72, 58 (1998).

${ }^{14}$ A. V. Uskov, F. Adler, H. Schweizer, and M. H. Pilkuhn, J. Appl. Phys. 81, 7895 (1997)

${ }^{15}$ A. V. Uskov, Y. Boucher, J. Le Bihan, and J. McInerney, Appl. Phys. Lett. 73, 1499 (1998).

${ }^{16}$ T. W. Berg, S. Bischoff, I. Magnusdottir, and J. Mørk, IEEE Photonics Technol. Lett. 13, 541 (2001).

${ }^{17}$ W. Rideout, W. F. Sharfin, E. S. Koteles, M. O. Vassel, and B. Elman, IEEE Photonics Technol. Lett. 3, 784 (1991).

${ }^{18}$ T. Inoshita and H. Sakaki, Phys. Rev. B 56, R4355 (1997).

${ }^{19}$ I. Magnusdottir, A. Uskov, S. Bischoff, B. Tromborg, J. Mørk, R. Ferreira, and G. Bastard, OSA Trends Opt. Photonics Ser. 74, 180 (2002).

${ }^{20}$ L. D. Landau and E. M. Lifschitz, Quantum Mechanics (ButterworthHeinemann, Oxford, U.K., 1977).

${ }^{21}$ V. M. Fomin, S. N. Klimin, V. N. Gladilin, and J. T. Devreese, J. Lumin. 87-89, 330 (2000)

${ }^{22}$ T. Takagahara, Phys. Rev. B 60, 2638 (1999).

${ }^{23}$ A. V. Uskov, A.-P. Jauho, B. Tromborg, J. Mørk, and R. Lang, Phys. Rev. Lett. 85, 1516 (1999)

${ }^{24}$ S. Hameau, Y. Guldner, O. Verzelen, R. Ferreira, G. Bastard, J. Zeman, A. Lemaitre, and J. M. Gérard, Phys. Rev. Lett. 83, 4152 (1999).

${ }^{25}$ C. Y. Tsai, C. Y. Tsai, Y. H. Lo, and L. F. Eastman, IEEE J. Quantum Electron. 31, 2148 (1995).

${ }^{26}$ B. K. Ridley, Quantum Processes in Semiconductors (Clarendon, Oxford, U.K., 1988).

${ }^{27}$ C. Cohen-Tannoudji, J. Dupont-Roc, and G. Grynberg, Atom-Photon Interactions (Wiley, New York, 1992).

${ }^{28}$ K. Zhang, J. Falta, Th. Schmidt, Ch. Heyn, G. Materlik, and W. Hansen, Pure Appl. Chem. 72, 199 (2000).

${ }^{29}$ C. Pryor, Phys. Rev. B 57, 7190 (1998).

${ }^{30}$ L. I. Schiff, Quantum Mechanics (McGraw-Hill, Singapore, 1968).

${ }^{31}$ V. I. Klimov, Ch. J. Schwarz, D. W. McBranch, C. A. Leatherdale, and M. G. Bawendi, Phys. Rev. B 60, R2177 (1999).

${ }^{32}$ V. I. Klimov, A. A. Mikhailovsky, S. Xu, A. Malko, J. A. Hollingsworth, C. A. Leatherdale, H.-J. Eisler, and M. G. Bawendi, Science 290, 314 (2000).

${ }^{33}$ M. Grundmann, O. Stier, and D. Bimberg, Phys. Rev. B 52, 11969 (1995).

${ }^{34}$ L. V. Asryan and R. A. Suris, Appl. Phys. Lett. 74, 1215 (1999). 